\title{
Medulloblastoma: clinicopathological parameters, risk stratification, and survival analysis of immunohistochemically validated molecular subgroups
}

\author{
Asmaa Mustafa Eid and Nehal Abd El-Ghaffar Heabah ${ }^{*}$ (1)
}

\begin{abstract}
Background: Medulloblastoma (MB) is a heterogeneous disease, displaying distinct genetic profiles with specific molecular subgroups. This study aimed to validate MB molecular subgrouping using surrogate immunohistochemistry and associate molecular subgroups, histopathological types, and available clinicopathological parameters with overall survival (OS) and progression-free survival (PFS) of MB patients. This study included $40 \mathrm{MBs}$; immunohistochemical staining, using $\beta$-catenin and GRB2-Associated Binding Protein 1 (GAB1) antibodies, was used to classify MB cases into wingless signaling activated (WNT), sonic hedgehog (SHH), and non-WNT/SHH molecular subgroups. Nuclear morphometric analysis (for assessment of degree of anaplasia) and Kaplan-Meier survival curves were done.
\end{abstract}

Results: MB cases were classified into WNT (10\%), SHH (30\%), and non-WNT/SHH (60\%) subgroups. Histopathological types differed significantly according to tumor location $(p<0.001)$, degree of anaplasia $(p=0.014)$, molecular subgroups $(p<0.001)$, and risk stratification $(p=0.008)$. Molecular subgroups differed significantly in age distribution ( $p=0.031)$, tumor location $(p<0.001)$, histopathological variants $(p<0.001)$, and risk stratification $(p<$ 0.001). OS was $77.5 \%$ and $50 \%$ after 1 and 2 years, while PFS was $65 \%$ and $27.5 \%$ after 1 and 2 years, respectively. OS and PFS were associated significantly with histopathological variants $(p<0.001$ and 0.001$)$, molecular subgroups $(p=0.012$ and 0.005$)$, and risk stratification ( $p<0.001$ and $<0.001)$, respectively.

Conclusions: Medulloblastoma classification based on molecular subgroups, together with clinicopathological indicators, mainly histopathological types; accurately risk stratifies MB patients and predicts their survival.

Keywords: Medulloblastoma, Molecular classification, $\beta$-catenin, GAB1, Histological types, Risk stratification, Overall survival, Progression-free survival

\section{Background}

Medulloblastoma (MB) is the most prevalent malignant pediatric brain tumor and accounts for up to $10 \%$ of childhood brain cancers. Advances in genome-wide analysis and gene transcription revealed that medulloblastomas are heterogeneous tumors, consisting of distinct molecular subgroups; each has a unique genomic profile

\footnotetext{
*Correspondence: nehalghaffar@yahoo.com; nehal.heaba@med.tanta.edu.eg
} Pathology Department, Faculty of Medicine, Tanta University, Tanta, Egypt [wingless signaling activated (WNT), sonic hedgehog (SHH), and non-WNT/non-SHH that further includes group 3 and group 4 medulloblastomas]. This molecular classification suggests different cellular origins with variable driving mutations [1].

Molecular classification based on DNA transcription and genome analysis is expensive and difficult for routine performance, especially in developing centers. Owing to its great importance in clinical practice, incorporation of this molecular classification into routine 
pathologic MBs evaluation is a must. Few studies used more simple techniques as fluorescence in situ hybridization (FISH), and immunohistochemistry (IHC), as surrogate methods for molecular subgrouping. Such techniques are easily applicable and provide reliable results on routinely processed formalin-fixed paraffinembedded (FFPE) specimens [1].

$\beta$-catenin and GAB1 antibodies can be used to classify $\mathrm{MB}$ into its three main molecular subgroups (WNT, $\mathrm{SHH}$, and non-WNT/SHH) [2].

WNT/ $\beta$-catenin signaling pathway regulates a wide range of vital cellular functions including cellular proliferation, differentiation, genetic stability, apoptosis, and tissue renewal. Aberrations in this pathway are implicated in several human malignancies including colonic carcinoma, breast carcinoma, adrenocortical tumor, melanoma, high-grade glioma, and MB. WNT subgroup of medulloblastoma (10-15\% of MB) comprises almost classic histology, rarely large cell/anaplastic (LCA) phenotype, but never desmoplastic/nodular $(\mathrm{D} / \mathrm{N})$ variant [3].

GAB1 (GRB2-Associated Binding Protein 1) belongs to Gab family and is believed to be a unique marker of SHH medulloblastoma subgroup [4]. SHH medulloblastoma subgroup (28-30\% of MB) includes desmoplastic nodular $(\mathrm{D} / \mathrm{N})$ morphology, a minority of classic variant, and less commonly LCA phenotype [5].

Non-WNT/SHHMB subgroup is molecularly defined by overexpression of MYC gene, and further subclassified into group 3 and group 4. Group 3 (25-28\% of all $\mathrm{MB}$ ) is the most aggressive MBs with a grave prognosis and a high metastatic rate at diagnosis. Classic and LCA medulloblastomas are the only histological variants encountered. Group 4 (40-45\% of all MB) shows a high incidence of chromosomal copy number variations. Classic histology is the most predominant, while LCA medulloblastomas are less commonly encountered in group $4 \mathrm{MB}[6,7]$.

The current work aimed to validate $\mathrm{MB}$ molecular subgrouping using surrogate $\mathrm{IHC}$, and associate these molecular subgroups, histopathological types, and available clinicopathological parameters with overall survival (OS) and progression-free survival (PFS) of MB patients.

\section{Methods}

This study was carried out on 40 cases of MB, diagnosed over 8 years (2010-2017) at Pathology Department, Faculty of Medicine, Tanta University, and private laboratories. This study was carried out during the period from May 2019 to August 2020. Cases were of adequate FFPE tissue blocks, complete clinical data including age at diagnosis, sex, tumor location, computerized tomography $(\mathrm{CT})$ mass size, metastasis at diagnosis $(\mathrm{M} 0$ or $\mathrm{M}+)$, type of surgery, size of residual mass after surgery, received postoperative radiation and/or chemotherapy, and complete follow-up data (recurrence and/or death). The current study was conducted after obtaining the approval from research ethics committee, Faculty of Medicine, Tanta University (reference\# 34152). Informed written consent was obtained from involved participation in the study.

\section{Methodology \\ Histopathological examination}

MB cases were classified histologically into classic, desmoplastic/nodular (D/N), and large cell/anaplastic (LCA) MBs, according to the 2016 WHO classification of tumors of the central nervous system (CNS) [5].

Degree of anaplasia Anaplasia in MBs was graded into a four tired scheme: no, slight, moderate, or severe anaplasia, according to four features: (a) enlarged nuclear size; (b) increased mitotic figures; (c) numerous apoptotic bodies; and (d) high pleomorphism with conspicuous nucleoli (large cell type) or pleomorphic crowded cells with frequent molding (anaplastic type). LCA variants were identified according to the presence of severe or even moderate anaplastic features even in a focal manner [8].

\section{Nuclear morphometric analysis for degree of anaplasia}

For histomorphometric analysis, hematoxylin and eosin (H\&E) stained sections were examined under a light microscope. Ten different non-overlapping randomly selected fields from each slide were examined at a magnification $(\times 400)$. The degree of anaplasia of MB cases was assessed by quantitative analysis of the histological photomicrographs for nuclear size (measured by nuclear perimeter in microns - arc length of a nuclear boundary [9], using an image analysis software (Image J; 1.52p software 32, NIH, USA)).

\section{Immunohistochemical analysis}

For clinical purposes, Taylor et al. recommended immunohistochemical use of $\beta$-catenin, GAB1 antibodies, to classify MBs into three molecular subgroups: WNT, $\mathrm{SHH}$, and non-WNT/SHH [2]. Formalin-fixed paraffinembedded tissue blocks were cut into $5-\mu \mathrm{m}$ sections. After processing with xylene, graded ethanol solutions, and $3 \% \mathrm{H}_{2} \mathrm{O}_{2}$ for $10 \mathrm{~min}$, antigen retrieval was performed in $0.05 \mathrm{M}$. citrate buffer $(\mathrm{pH}=6.0)$ at $100^{\circ} \mathrm{C}$ for 5-10 min followed by blocking in goat serum for $10 \mathrm{~min}$. Deparaffinization and antigen retrieval were performed in a Dako PT Link unit. Both high and low pH EnVision TM FLEX Target Retrieval Solutions were used at $97^{\circ} \mathrm{C}$ for $20 \mathrm{~min}$.

Dako automated immune-stainer (Link 48) was used for immunostaining using $\beta$-catenin antibody, a mouse monoclonal antibody (clone 12F7, sc-59737, Santa Cruz 
Biotechnology, Inc., USA) and GAB1 antibody, a mouse monoclonal antibody (clone H-7: sc-13319, Santa Cruz Biotechnology, Inc., USA). The slides were incubated with primary antibodies for $20-30 \mathrm{~min}$, following treatment with a peroxidase-blocking reagent for $5 \mathrm{~min}$. Horseradish peroxidase (HRP) reagent was added for 20 min and diaminobenzidine (DAB) chromogen solution for $10 \mathrm{~min}$. Meyer's hematoxylin was applied for counterstaining.

\section{Assessment of $\beta$-catenin IHC results}

Nuclear $\beta$-catenin immunoreactivity in $\geq 5 \%$ of tumor cells was considered positive. Either nuclear $\beta$-catenin immunoreactivity in $<5 \%$ of tumor cells or cytoplasmic positivity were considered negative for $\beta$-catenin expression (entire $\beta$-catenin negativity is exceptional in $\mathrm{MB}$ ) [10]. Positive control included specimens of normal colon and colonic carcinoma. Negative control was performed by replacing the primary antibody with phosphate-buffered saline (PBS).

\section{Assessment of GAB1 IHC results}

GAB1 positivity was detected as cytoplasmic staining in $\geq 30 \%$ of tumor cells, meanwhile percentage of positive cells $<30 \%$ was regarded as negative [11]. Positive control was tonsillar tissue. Negative control was performed by replacing the primary antibody with PBS.

Synaptophysin, NeuN, and INI immunohistochemistry further confirmed the diagnosis of anaplastic MB with rhabdoid features. IHC revealed positive cytoplasmic and nuclear reactions for synaptophysin and NeuN, respectively, as well as intact nuclear expression of integrase interactor 1 (INI 1) (to exclude atypical teratoid rhabdoid tumor $[\mathrm{AT} / \mathrm{RT}])$.

\section{Risk stratification}

Patients were classified into standard and high-risk based on age at diagnosis ( $>3$ or $<3$ years), size of postoperative residual mass (maximum cross-sectional area $<1.5$ and $>1.5 \mathrm{~cm}^{2}$ ), histology, and metastatic disease at diagnosis (M0 or $\mathrm{M}+)$ [12].

\section{Statistical analysis}

Statistical analysis was performed using the IBM SPSS software package version 20.0. (Armonk, NY: IBM Corp). Data were expressed as frequencies for categorical variables, and continuous variables were expressed as mean $\pm \mathrm{SD}$ or median and range. The KolmogorovSmirnov test was used to verify the normality of distribution of variables. For comparing categorical variables, chi-square $\left(x^{2}\right)$ and Monte Carlo (MC) tests were applied.

Survival analyses [overall survival (OS) and progression-free survival (PFS)] were performed. OS was the time from date of diagnosis to death or the date of last follow-up. PFS was the time interval from date of surgery to the date of progression or relapse. KaplanMeier survival curves were done for the significant relation with OS and PFS. Kaplan-Meier survival analysis with number at risk was done using the MedCalc Statistical Software version 18.9.1 (MedCalc Software bvba, Ostend, Belgium). $P$ value $<0.05$ was considered statistically significant.

\section{Results \\ Clinical characteristics}

The current study included $40 \mathrm{MB}$ patients. Their clinical data are summarized in Table 1.

Histopathological features, nuclear morphometric analysis for degree of anaplasia, and molecular subgroups

Based on microscopic evaluation of $40 \mathrm{MBs}, 16$ cases (40\%) were of LCA histology, 14 cases of classic histology (35\%), and 10 cases were D/N MB (25\%).

\section{Morphometric analysis for degree of anaplasia}

Based on combined histopathological examination and image analysis, 18 cases (45\%) showed severe anaplastic features, 15 cases (37.5\%) showed moderate anaplasia, and 7 cases (17.5\%) showed slight anaplasia. Mean nuclear perimeter for different histopathological types was as follows: $44.529 \mu \mathrm{m}$ for $\mathrm{D} / \mathrm{N}, 46.996 \mu \mathrm{m}$ for classic, and $62.237 \mu \mathrm{m}$ for LCA phenotype.

\section{Molecular subgrouping}

Based on IHC staining results, the WNT subgroup (nuclear $\beta$-catenin positivity, cytoplasmic GAB1 negativity) represented $10 \%$ of cases; $\mathrm{SHH}$ subgroup (nuclear $\beta$ catenin negativity, cytoplasmic GAB1 positivity) represented $30 \%$ of cases, and non-WNT/SHH (both nuclear $\beta$-catenin, cytoplasmic GAB1 negativity) represented $60 \%$ of cases (Table 2, Fig. 1).

\section{Relation of histopathological types to clinicopathological parameters}

The histopathological types differed significantly according to tumor location ( $p$ value $<0.001$ ), degree of anaplasia $(p$ value $=0.014)$, molecular subgroups $(p$ value $<$ $0.001)$, and risk stratification ( $p$ value $=0.008)($ Table 3$)$.

The majority of classic MBs and LCA were diagnosed at pediatric age (78.6\% and $75 \%$, respectively); $\mathrm{D} / \mathrm{N}$ MBs were distributed among all age groups (however, no significant relation was detected between histopathological types and age of the patients). Most of classic and LCA MBs were located at the midline $(92.9 \%$ and $81.3 \%$, respectively), whereas $90 \%$ of $\mathrm{D} / \mathrm{N}$ cases were located laterally at the cerebellar hemispheres. 
Table 1 Clinical characteristics of the studied cases

\begin{tabular}{|c|c|}
\hline & No. (\%) \\
\hline \multicolumn{2}{|l|}{ Age in years } \\
\hline$<3$ (infants) & $8(20 \%)$ \\
\hline 3-16 (pediatric age) & $27(67.5 \%)$ \\
\hline$>16$ (adults) & $5(12.5 \%)$ \\
\hline Mean \pm SD & $7.7 \pm 6.2$ \\
\hline Median (min.-max.) & $5(1.8-26)$ \\
\hline \multicolumn{2}{|l|}{ Sex } \\
\hline Male & $22(55 \%)$ \\
\hline Female & $18(45 \%)$ \\
\hline \multicolumn{2}{|l|}{ Location of the mass } \\
\hline Midline & $27(67.5 \%)$ \\
\hline Lateral & $13(32.5 \%)$ \\
\hline \multicolumn{2}{|c|}{ Computerized tomography $(\mathrm{CT})$ mass size } \\
\hline$<3 \mathrm{~cm}$ & $21(52.5 \%)$ \\
\hline$>3 \mathrm{~cm}$ & $19(47.5 \%)$ \\
\hline \multicolumn{2}{|l|}{ Type of surgery } \\
\hline Gross-total resection & $6(15 \%)$ \\
\hline Near-total resection & $10(25 \%)$ \\
\hline Sub-total resection & $24(60 \%)$ \\
\hline \multicolumn{2}{|l|}{ Residual size after surgery } \\
\hline$<1.5 \mathrm{~cm}^{2}$ & $18(45 \%)$ \\
\hline$>1.5 \mathrm{~cm}^{2}$ & $22(55 \%)$ \\
\hline \multicolumn{2}{|l|}{ Metastasis at diagnosis } \\
\hline M0 & $21(52.5 \%)$ \\
\hline $\mathrm{M}+$ & $19(47.5 \%)$ \\
\hline \multicolumn{2}{|l|}{ Postoperative protocol } \\
\hline No therapy & $3(7.5 \%)$ \\
\hline Radiation therapy & $13(32.5 \%)$ \\
\hline Radiation plus chemotherapy & $24(60 \%)$ \\
\hline \multicolumn{2}{|l|}{ Risk stratification } \\
\hline Standard risk & $18(45 \%)$ \\
\hline High risk & $22(55 \%)$ \\
\hline \multicolumn{2}{|l|}{ Recurrence rate } \\
\hline No & $11(27.5 \%)$ \\
\hline Yes & $29(72.5 \%)$ \\
\hline \multicolumn{2}{|l|}{ Death } \\
\hline Survival & $20(50 \%)$ \\
\hline Death & 20 (50\%) \\
\hline
\end{tabular}

Half of classic histology showed moderate anaplasia, while $75 \%$ of LCA cases showed marked anaplasia. Regarding the molecular subgroups, $78.6 \%$ of classic histology was of non-WNT/SHH profile, $21.4 \%$ of WNT type. D/N cases were exclusive of $\mathrm{SHH}$ type (100\%).
Table 2 Histopathological, IHC results, and molecular subgroups

\begin{tabular}{ll}
\hline & No. (\%) \\
\hline Histopathological types & $14(35 \%)$ \\
Classic medulloblastoma & $10(25 \%)$ \\
Lesmoplastic/nodular medulloblastoma & $16(40 \%)$ \\
Degree of anaplasia & \\
Slight anaplasia & $7(17.5 \%)$ \\
Moderate anaplasia & $15(37.5 \%)$ \\
Severe anaplasia & $18(45 \%)$ \\
$\beta$-catenin expression & \\
Positive nuclear expression & $4(10 \%)$ \\
Negative both nuclear and cytoplasmic expression & $4(10 \%)$ \\
Cytoplasmic expression & $32(80 \%)$ \\
GAB1 expression & \\
Negative & $28(70 \%)$ \\
Positive & $12(30 \%)$ \\
Molecular subgroups & \\
WNT & $4(10 \%)$ \\
SHH & $12(30 \%)$ \\
Non-WNT/SHH & $24(60 \%)$ \\
\hline
\end{tabular}

LCA histology showed mostly (81.3\%) non-WNT/SHH profile.

Considering risk stratification, $50 \%$ and $80 \%$ of classic and $\mathrm{D} / \mathrm{N}$ cases were of standard-risk group, respectively, while $81.3 \%$ of LCA cases were of high-risk group.

\section{Relation of molecular subgroups to clinicopathological parameters}

The molecular subgroups differed significantly in age distribution ( $p$ value $=0.031)$, tumor location $(p$ value $<$ $0.001)$, histopathological variants $(p$ value $<0.001)$, and risk stratification $(p$ value $<0.001)$. No significant relation was detected between the molecular subgroups and degree of anaplasia of the studied cases (Table 4).

Regarding WNT tumors, 75\% of WNT tumors were detected among pediatric age (3-16 years) and were not seen in infants. They were all located in the midline and were mainly of classic histology (75\%). All WNT cases showed standard risk of stratification.

SHH tumors were detected among all age groups; 50\% of cases were detected among 3-16 years, 33.3\% of cases were diagnosed in adults ( $>16$ years) and $16.7 \%$ of cases were diagnosed in infants (<3 years). Most $\mathrm{SHH}$ MBs were laterally located (91.7\%). It included D/N (83.3\%) as well as LCA (16.7\%) phenotypes; $83.3 \%$ of SHH cases showed standard-risk. 


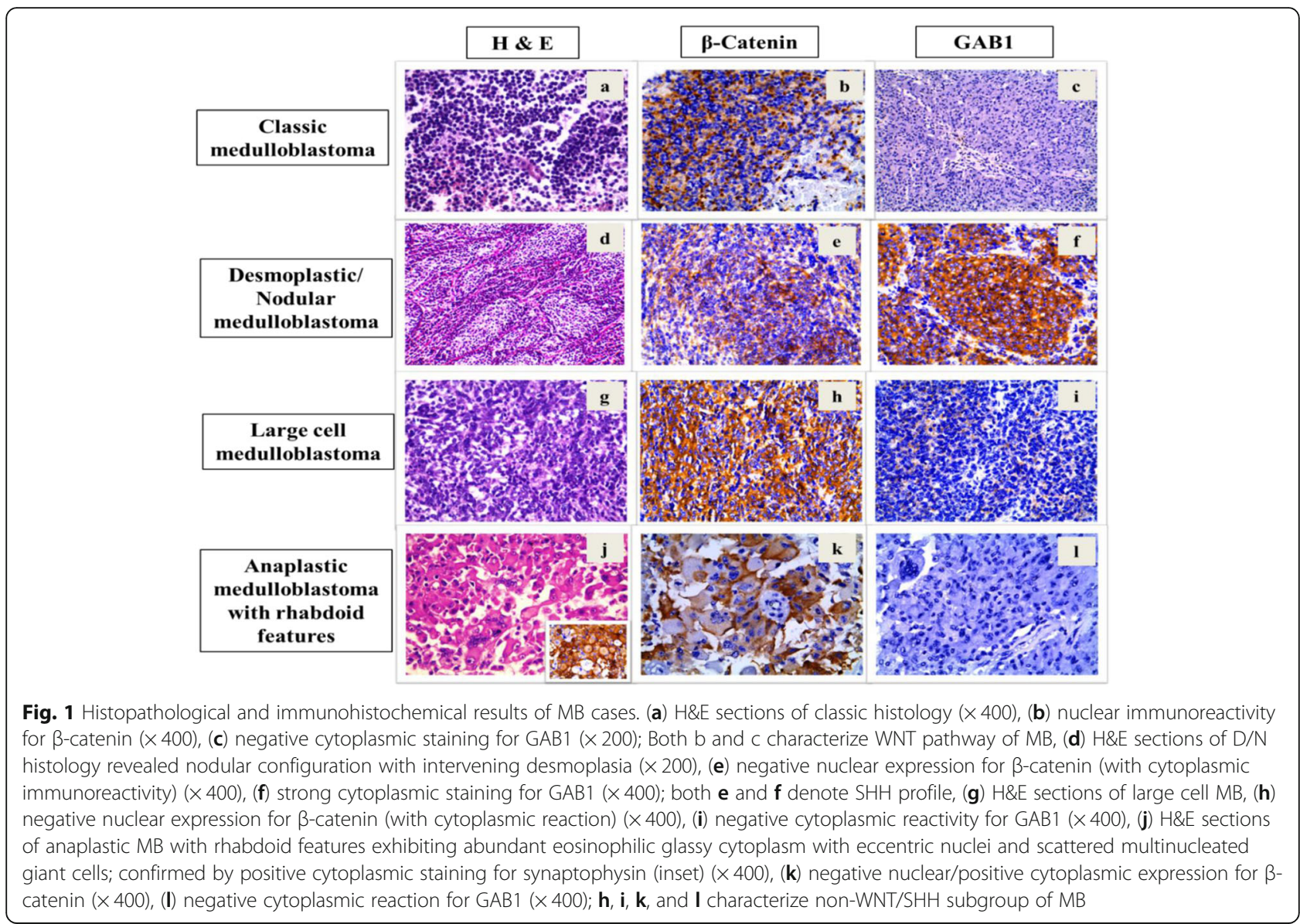

Non-WNT/SHH MBs were predominantly diagnosed in the pediatric age group (75\%), 22 cases (91.7\%) were located at midline; LCA and classic histology (54.2\%, $45.8 \%$, respectively) were seen in this subgroup. The majority of non-WNT/SHH MBs were high-risk tumors.

\section{Survival analysis}

The follow-up period was 2 years ( 24 months). The OS was $77.5 \%$ and $50 \%$ after 1 and 2 years, respectively, with a mean of 19.1 months and median of 24 months (95\% CI, 17.1-21.1). The PFS was $65 \%$ and $27.5 \%$ after 1 and 2 years, respectively, with a mean of 15.83 months and median of 17 months (95\% CI, 13.6-18.1) (Table 5, Fig. 2).

\section{Relations of OS and PFS to different clinicopathological parameters}

Kaplan-Meier curves revealed that both OS and PFS associated significantly with histopathological variants $(p$ value $<0.001$ and 0.001 ), molecular subgroups ( $p$ value $=$ 0.012 and 0.005$)$, and risk stratification ( $p$ value $<0.001$ and $<0.001$ ), respectively. MBs of LCA histology exhibited the worst OS and PFS (18.8\% and $12.5 \%$, respectively). Among the molecular subgroups, WNT had the best outcome with excellent PFS (100\%), and the nonWNT/SHH showed the worst OS (33.3\%). Both OS and PFS were poor with high-risk group patients $(22.7 \%$ and 9.1\%, respectively). Furthermore, PFS was significantly associated with the degree of cellular anaplasia, being worst with severe anaplasia $(5.6 \%)(p$ value $=0.003)$ (Table 6, Figs. 3 and 4).

\section{Discussion}

Medulloblastoma patients, sharing the same WHO histopathological type, have peculiar genetic backgrounds and different prognoses. Therefore, diagnosis of $\mathrm{MB}$ requires a combined routine histopathological evaluation (including microscopic type and histological grade of malignancy "WHO Grade IV") with additional molecular features, to give an accurate integrated (or the so-called "layered") diagnosis, and to allow a more refined risk stratification [5].

In the current study, MB histopathological types included classic (35\%), D/N (25\%), and LCA (40\%) of MBs. These histopathological types were significantly associated with tumor location, degree of anaplasia, molecular subgroups, and risk stratification. Most LCA cases showed marked degree anaplasia. Most of the 
Table 3 Relation of histopathological types to clinicopathological parameters

\begin{tabular}{|c|c|c|c|c|}
\hline & \multicolumn{3}{|l|}{ Histopathological types } & \multirow[t]{2}{*}{$P$ value } \\
\hline & $\begin{array}{l}\text { Classic medulloblastoma } \\
(n=14)\end{array}$ & $\begin{array}{l}\text { Desmoplastic /Nodular } \\
\text { medulloblastoma }(n=10)\end{array}$ & $\begin{array}{l}\text { Large cell/anaplastic } \\
\text { medulloblastoma }(n=16)\end{array}$ & \\
\hline \multicolumn{5}{|l|}{$\begin{array}{l}\text { Age group } \\
\text { (years) }\end{array}$} \\
\hline$<3$ (infants) & $3(21.4 \%)$ & $2(20 \%)$ & $3(18.8 \%)$ & $M C, p=0.073$ \\
\hline $\begin{array}{l}\text { 3-16 (pediatric } \\
\text { age) }\end{array}$ & $11(78.6 \%)$ & $4(40 \%)$ & $12(75 \%)$ & \\
\hline$>16$ (adults) & $0(0 \%)$ & $4(40 \%)$ & $1(6.3 \%)$ & \\
\hline \multicolumn{5}{|l|}{ Sex } \\
\hline Male & $9(64.3 \%)$ & $4(40 \%)$ & $9(56.3 \%)$ & $p=0.495$ \\
\hline Female & $5(35.7 \%)$ & $6(60 \%)$ & 7 (43.8\%) & \\
\hline \multicolumn{5}{|l|}{$\begin{array}{l}\text { Location of the } \\
\text { mass }\end{array}$} \\
\hline Midline & $13(92.9 \%)$ & $1(10 \%)$ & $13(81.3 \%)$ & $M C, p<0.001^{*}$ \\
\hline Lateral & $1(7.1 \%)$ & $9(90 \%)$ & $3(18.8 \%)$ & \\
\hline \multicolumn{5}{|l|}{$\begin{array}{l}\text { Degree of } \\
\text { anaplasia }\end{array}$} \\
\hline Slight anaplasia & $4(28.6 \%)$ & $3(30 \%)$ & $0(0 \%)$ & $M C, p=0.014^{*}$ \\
\hline $\begin{array}{l}\text { Moderate } \\
\text { anaplasia }\end{array}$ & $7(50 \%)$ & $4(40 \%)$ & $4(25 \%)$ & \\
\hline $\begin{array}{l}\text { Severe } \\
\text { anaplasia }\end{array}$ & $3(21.4 \%)$ & $3(30 \%)$ & $12(75 \%)$ & \\
\hline \multicolumn{5}{|l|}{$\begin{array}{l}\text { Molecular } \\
\text { subgroups }\end{array}$} \\
\hline WNT & $3(21.4 \%)$ & $0(0 \%)$ & $1(6.3 \%)$ & $M C, p<0.01^{*}$ \\
\hline $\mathrm{SHH}$ & $0(0 \%)$ & $10(100 \%)$ & $2(12.5 \%)$ & \\
\hline Non-WNT/SHH & $11(78.6 \%)$ & $0(0 \%)$ & $13(81.3 \%)$ & \\
\hline \multicolumn{5}{|l|}{$\begin{array}{l}\text { Risk } \\
\text { stratification }\end{array}$} \\
\hline Standard risk & $7(50 \%)$ & $8(80 \%)$ & $3(18.8 \%)$ & $p<0.008^{*}$ \\
\hline High risk & $7(50 \%)$ & $2(20 \%)$ & $13(81.3 \%)$ & \\
\hline
\end{tabular}

MC Monte Carlo

*Statistically significant at $p \leq 0.05$

classic and $\mathrm{D} / \mathrm{N}$ cases in our results were of standard-risk group, while most of LCA cases were of high-risk group. These results were in harmony with Ellison et al., who reported that high-risk disease was associated with LCA phenotype and metastasis at diagnosis $(\mathrm{M}+)$ [1]. Also, Jiang et al. noted that LCA histology was an independent risk factor with a grave prognosis and claimed that such diagnoses should require intensive treatments [13].

In our results, no significant relation could be detected between the histopathological types and age of the patients. However, Al-Halabi et al. supposed that D/N medulloblastomas contributed to most MBs in infancy and adulthood, sparing the pediatric period [14].

The molecular subgroups in our study differed significantly in age distribution, tumor location, histopathological variants, and risk stratification. WNT tumors represented $10 \%$ of cases; most of them were detected among pediatric age (3-16 years) and were mainly of classic histology. Our results matched those of Ellison et al., who stated that WNT subgroup was the rarest (10\% of all MBs) and that WNT medulloblastomas were almost all classic tumors ( $81 \%$ of their cases) and presented between the ages of 6-12 years [1]. According to Pietsch et al., WNT MBs mostly occur in children older than 3 years or teenagers [15]. Taylor et al. stated that WNT medulloblastomas rarely have LCA histology, but even with this histology, they showed an excellent prognosis [2].

In the current study, all WNT MBs located at midline, which was in agreement with Pietsch et al., who declared that WNT tumors were located mainly in the midline (their cell of origin derives from the lower rhombic lip) [15]. Gibson et al. also found that among MB cases in a 
Table 4 Relation of molecular subgroups to clinicopathological parameters

\begin{tabular}{|c|c|c|c|c|}
\hline & \multicolumn{3}{|c|}{ Molecular subgroups } & \multirow[t]{2}{*}{$P$ value } \\
\hline & WNT $(n=4)$ & $\mathrm{SHH}(n=12)$ & Non-WNT/SHH $(n=24)$ & \\
\hline \multicolumn{5}{|l|}{ Age group (years) } \\
\hline$<3$ (infants) & $0(0 \%)$ & $2(16.7 \%)$ & $6(25 \%)$ & \multirow[t]{3}{*}{$M C, p=0.031^{*}$} \\
\hline 3-16 (pediatric age) & $3(75 \%)$ & $6(50 \%)$ & $18(75 \%)$ & \\
\hline$>16$ (adults) & $1(25 \%)$ & $4(33.3 \%)$ & $0(0 \%)$ & \\
\hline \multicolumn{5}{|l|}{ Sex } \\
\hline Male & $1(25 \%)$ & $5(41.7 \%)$ & $16(66.7 \%)$ & \multirow[t]{2}{*}{$M C, p=0.183$} \\
\hline Female & $3(75 \%)$ & $7(58.3 \%)$ & $8(33.3 \%)$ & \\
\hline \multicolumn{5}{|l|}{ Location of the mass } \\
\hline Midline & $4(100 \%)$ & $1(8.3 \%)$ & $22(91.7 \%)$ & \multirow[t]{2}{*}{$M C, p<0.001^{*}$} \\
\hline Lateral & $0(0 \%)$ & $11(91.7 \%)$ & $2(8.3 \%)$ & \\
\hline \multicolumn{5}{|l|}{ Histopathological types } \\
\hline Classic medulloblastoma & $3(75 \%)$ & $0(0 \%)$ & $11(45.8 \%)$ & \multirow[t]{3}{*}{$M C, p<0.001^{*}$} \\
\hline Desmoplastic/nodular medulloblastoma & $0(0 \%)$ & $10(83.3 \%)$ & $0(0 \%)$ & \\
\hline Large cell/anaplastic medulloblastoma & $1(25 \%)$ & $2(16.7 \%)$ & $13(54.2 \%)$ & \\
\hline \multicolumn{5}{|l|}{ Degree of anaplasia } \\
\hline Slight anaplasia & $2(50 \%)$ & $3(25 \%)$ & $2(8.3 \%)$ & \multirow[t]{3}{*}{$M C, p=0.255$} \\
\hline Moderate anaplasia & $1(25 \%)$ & $5(41.7 \%)$ & $9(37.5 \%)$ & \\
\hline Severe anaplasia & $1(25 \%)$ & $4(33.3 \%)$ & $13(54.2 \%)$ & \\
\hline \multicolumn{5}{|l|}{ Risk stratification } \\
\hline Standard risk & $4(100 \%)$ & $10(83.3 \%)$ & $4(16.7 \%)$ & \multirow[t]{2}{*}{$M C, p<0.001^{*}$} \\
\hline High risk & $0(0 \%)$ & $2(16.7 \%)$ & $20(83.3 \%)$ & \\
\hline
\end{tabular}

MC Monte Carlo

*Statistically significant at $p \leq 0.05$

mouse model study, WNT subgroup arose from the midline of the brain stem [16].

In our study, all WNT cases exhibited standard-risk stratification. Ellison et al. reported that some cases with high-risk features (including LCA morphology or $\mathrm{M}+$ ) showed favorable outcomes, interestingly, when associated with WNT profile [1].

SHH tumors, in this study, enclosed $30 \%$ of cases. The same finding was detected by Northcott et al. and Pietsch et al., as SHH subgroup represented $30 \%$ of their MBs [5, 17]. $\mathrm{SHH}$ tumors in this study were detected in all age groups, mainly among pediatric age group (3-16 years). In contrast, Zhukova et al. and Pietsch et al. found that SHH tumors had a bimodal age distribution affecting both the infants and adults, sparing the pediatric period $[5,18]$. Kool et al. reported that $\mathrm{SHH}$ medulloblastomas were found in infants and adults and occurred much less frequently in patients aged 3-15 years [19].
In the current study, most SHH MBs were laterally located. Gibson et al. reported that these tumors derived from the cerebellar granular precursor cells of the external granular layer (originated laterally from the cerebellar hemispheres) [16].

All D/N MBs in this study were of SHH type. Pietsch et al. reported that $\mathrm{D} / \mathrm{N}$ variant was almost exclusive for SHH-MB, followed by classic and LCA subtypes [5]. Ellison et al. also stated that all desmoplastic tumors were included in the SHH pathway [1]. However, Taylor et al. reported that $\mathrm{SHH}$ medulloblastomas included both desmoplastic types and not desmoplastic/nodular types (up to 50\%) [2].

Non-WNT/SHH MBs (60\% of our cases), were predominantly diagnosed in the pediatric age group (3-16 years) and were located mainly at the midline. Most of its cases were LCA and classic MBs and were high-risk tumors. Cho et al. and Tamayo et al. reported that non-WNT/SHH MB constituted the most common molecular subgroup and

Table 5 Overall survival (OS) and progression-free survival (PFS) of the studied cases

\begin{tabular}{llllll}
\hline & Mean (months) & $\mathbf{9 5 \% ~ C l}$ & Median (months) & \% 1 year & \% 2 year (end of study) \\
\hline Overall survival (OS) & 19.1 & $17.1-21.1$ & 24 & $77.5 \%$ & $50 \%$ \\
Progression-free survival (PFS) & 15.83 & $13.6-18.1$ & 17 & $65 \%$ & $27.5 \%$ \\
\hline
\end{tabular}

Cl confidence interval 


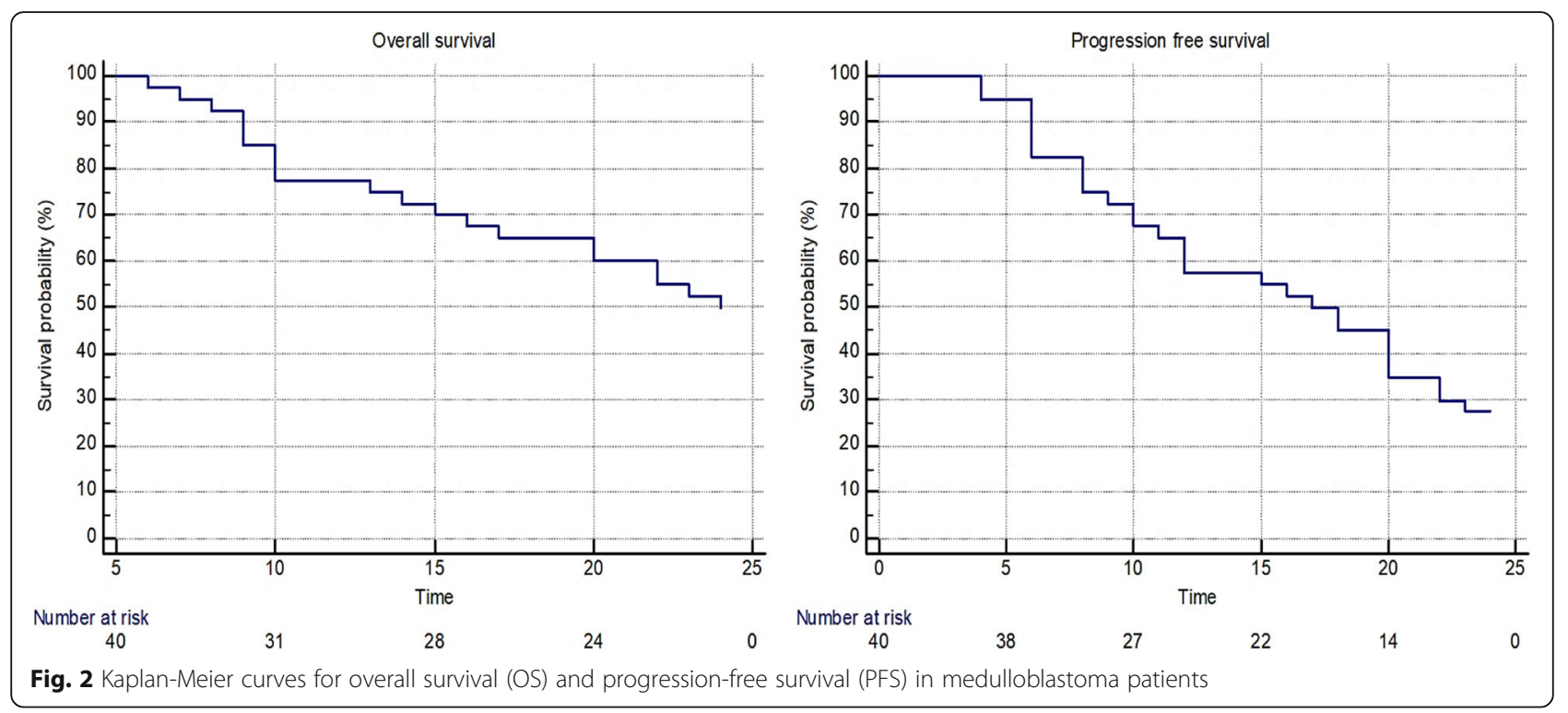

Table 6 Relation of overall survival (OS) and progression-free survival (PFS) to different clinicopathological parameters

\begin{tabular}{|c|c|c|c|c|c|c|c|c|}
\hline & \multicolumn{4}{|c|}{ Overall survival (OS) } & \multicolumn{3}{|c|}{ Progression-free survival (PFS) } & \multirow[b]{2}{*}{$P$ value } \\
\hline & Mean & Median & $\%$ End of study & $P$ value & Mean & Median & $\%$ End of study & \\
\hline \multicolumn{9}{|l|}{ Age group (years) } \\
\hline$<3$ (infants) & 18.38 & 20 & $50 \%$ & 0.363 & 13.75 & 11 & $25 \%$ & 0.182 \\
\hline 3-16 (pediatric age) & 18.44 & 22 & $44.4 \%$ & & 15.15 & 17 & $22.2 \%$ & \\
\hline$>16$ (adults) & 23.8 & & $80 \%$ & & 22.8 & & $60 \%$ & \\
\hline \multicolumn{9}{|l|}{ Histopathological types } \\
\hline Classic medulloblastoma & $21.93^{\#}$ & & $71.4 \%$ & $<0.001^{*}$ & $20.5^{\#}$ & 22 & $42.9 \%$ & $0.001^{*}$ \\
\hline Desmoplastic/nodular medulloblastoma & $23.1^{\#}$ & & $70 \%$ & & $18.7^{\#}$ & 20 & $30 \%$ & \\
\hline Large cell/anaplastic medulloblastoma & 14.13 & 10 & $18.8 \%$ & & 9.94 & 8 & $12.5 \%$ & \\
\hline \multicolumn{9}{|l|}{ Degree of anaplasia } \\
\hline Slight anaplasia & 22.86 & & $71.4 \%$ & 0.274 & $20.71^{\#}$ & & $57.1 \%$ & $0.003^{*}$ \\
\hline Moderate anaplasia & 19.53 & & $53.3 \%$ & & $18^{\#}$ & 20 & $40 \%$ & \\
\hline Severe anaplasia & 17.28 & 16 & $38.9 \%$ & & 12.11 & 11 & $5.6 \%$ & \\
\hline \multicolumn{9}{|l|}{ Molecular Subgroups } \\
\hline WNT & $24^{\#}$ & & $100 \%$ & $0.012^{*}$ & 24 & & $100 \%$ & $0.005^{*}$ \\
\hline $\mathrm{SHH}$ & $23.08^{\#}$ & & $66.7 \%$ & & $18.92^{\#}$ & 20 & $33.3 \%$ & \\
\hline Non-WNT/SHH & 16.29 & 15 & $33.3 \%$ & & $12.92^{\#}$ & 10 & $12.5 \%$ & \\
\hline \multicolumn{9}{|l|}{ Risk stratification } \\
\hline Standard risk & 23.61 & & $83.3 \%$ & $<0.001^{*}$ & 21.61 & 23.0 & $50 \%$ & $<0.001^{*}$ \\
\hline High risk & 15.41 & 14 & $22.7 \%$ & & 11.09 & 9.0 & $9.1 \%$ & \\
\hline
\end{tabular}

OS overall survival

"Statistically significant with large cell/anaplastic medulloblastoma

"Statistically significant with non-WNT/SHH

PFS progression-free survival

${ }^{\#}$ Statistically significant with large cell/anaplastic medulloblastoma

"Statistically significant with severe anaplasia

"Statistically significant with WNT 


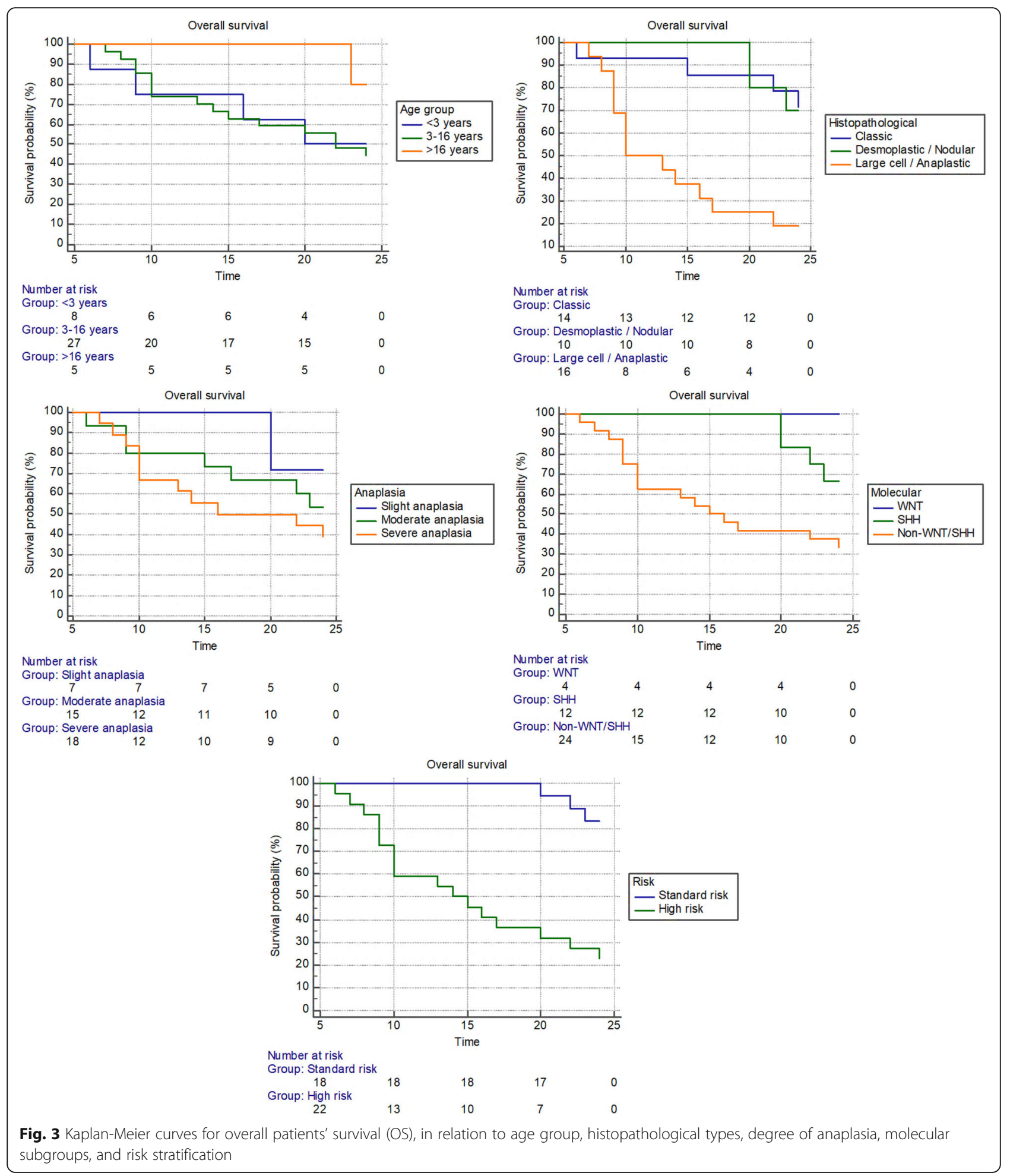

that the MBs of this group located in the midline filling the fourth ventricle $[6,7]$. According to their studies, two histologic variants are encountered in this group, classic and LCA. Also, they reported that non-WNT/SHH MBs were of high-risk group with dismal prognosis.
In this study, the 2-year OS was $50 \%$ and the 2-year PFS was 27.5\%; Tarbell et al. reported a higher 5-year OS (60\%) [20]. In our study, no association was detected between OS and age at diagnosis. However, Sirachainan et al. reported that the 5-year OS rate in children (3-16 years) was $60.6 \%$, 


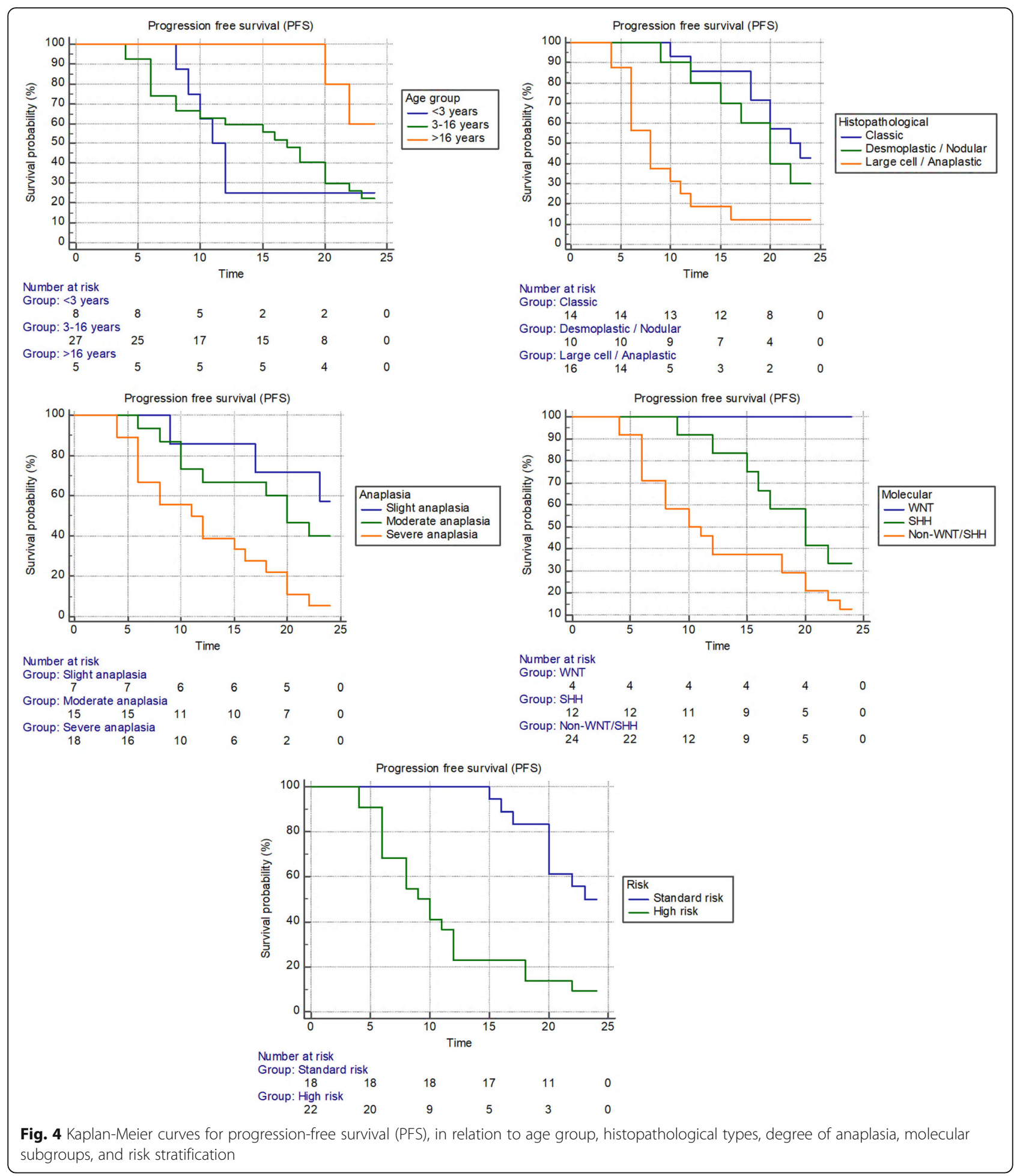

whereas children in a study by Nalita et al. had a $53.8 \%$ 5-year OS rate [21, 22]. Schwalbe et al. categorized $\mathrm{SHH}$ patients into two groups $\mathrm{SHH}$ infant and SHH child); which had a 58 and $48 \%$ 10-year OS, respectively [4].
The OS and PFS in our study, associated significantly with histopathological types, molecular subgroups, and risk stratification. Histologically, classic and D/N types showed nearly similar OS (71.4\% and 70\%, respectively), with PFS of $42.9 \%$ and 30\%, respectively. LCA histology 
exhibited the worst OS and PFS (18.8\% and 12.5\%, respectively). Similarly, Louis et al. reported that D/N variant exhibited the best prognosis, whereas, LCA variant had a poor prognosis [23]. Yet, Nalita et al. found no significant differences of survival rates between histological variants [22]. Gupta et al. suggested that MB histology could determine patients' outcomes (rather than other prognostic factors such as age, concurrent CNS involvement, visceral metastases, or time to relapse) [24].

In this work, patients with severe anaplasia showed significantly worse PFS (5.6\%), Giangaspero et al. also found that progression-free survival for MBs with severe anaplasia was significantly shorter than tumors with slight or moderate anaplastic features [25].

In our study, molecular subgroups were prognostically important, with significantly different survival rates. WNT tumors had the best outcome with excellent PFS, and non-WNT/SHH showed the worst and shortest OS (33.3\%). SHH medulloblastomas had an intermediate (66.7\%) OS. Ellison et al., Kool et al., Northcott et al., and Taylor et al. reported the best outcome and a high 5 -year OS ( 95\%) for the WNT subgroup, an intermediate $(75-80 \%)$ OS for SHH MBs, while the worst and shortest survival for non-WNT/SHH subgroup [1, 2, 17, 19]. Ramaswamy et al. also confirmed that the WNT subtype had the best clinical outcome, with a 5-year OS $>95 \%$ [26]. Cho et al. found that the SHH subgroup had intermediate prognosis, with a $70 \% 5$-year OS [6].

According to Thompson et al., the prognosis of WNT MB was excellent, even in the presence of poor outcome indicators as somatic TP53 mutation, incomplete resection, and/or metastatic disease at presentation [27]. Many studies explained that the good outcome of WNT subgroup was due to the presence of WNT antagonistic secretions that modifies the permeability of blood-brain barrier; allowing high penetrance of chemotherapeutic agents into the tumor site [28]. This could permit a less aggressive approach in treating WNT tumors [3].

In combination with clinical and pathological outcome indicators, molecular markers are not only prognostically important but would also facilitate the use of targeted therapies, such as GDC-0449, a novel SHH pathway inhibitor, particularly in infants and adults [1].

In the current study, both OS and PFS were poor with high-risk group patients (22.7\% and 9.1\%, respectively), while in the standard-risk group, the OS and PFS were $83.3 \%$ and $50 \%$, respectively. Nalita et al. also reported $84.4 \%$ and $42.8 \%$ OS rates of standard-risk and high-risk groups, respectively [22].

Tarbell et al., Ramaswamy et al., and Ramaswamy et al. reported higher 5-year survival rates (for high-risk MBs) reaching 60\% [20, 26, 29]. Sirachainan et al. reported OS rates of standard-risk and high-risk groups of $58-85 \%$ and $32-70 \%$, respectively [21]. Thompson et al. reported that patients with postsurgical residual tumor $>1.5 \mathrm{~cm}^{2}$ (an indicator of high-risk disease) had worse PFS and required aggressive treatment options [27].

Clinical trials should incorporate key molecular profiles, including subgroup information, genetic, cytogenetic, and epigenetic changes, of this diverse disease entity that can suggest precise patients' outcomes or predict rational treatment strategies [30].

The rarity of some WHO subtypes of MB; specifically, medulloblastoma with extensive nodularity, is considered a limitation of this study. Larger studies including all the histopathological types of medulloblastoma are recommended.

\section{Conclusions}

In conclusion, histopathological types, molecular subgroups (determined by $\beta$-catenin and GAB1 immunohistochemistry), and risk stratification are important prognosticators, and are associated with overall and progression-free survival of $\mathrm{MB}$ patients. Patients with the same pathological type of $\mathrm{MB}$ may have distinct genetic backgrounds and different prognosis. Advanced molecular testing is recommended to yield better results, confirm the current data and further classify each molecular subgroup.

\section{Abbreviations \\ Cl: Confidence interval; CNS: Central nervous system; CT: Computerized tomography; D/N: Desmoplastic/nodular; DAB: Diaminobenzidine; FFPE: Formalin-fixed paraffin-embedded; FISH: Fluorescence in situ hybridization; GAB1: GRB2-Associated Binding Protein 1; H\&E: Hematoxylin and eosin; HRP: Horseradish peroxidase; IHC: Immunohistochemistry; INI 1: Integrase interactor 1; LCA: Large cell/anaplastic; MB: Medulloblastoma; MC: Monte Carlo; OS: Overall survival; PFS: Progression-free survival; SHH: Sonic hedgehog; WHO: World Health Organization; WNT: Wingless signaling activated; $\times 2$ : Chi-square}

\section{Acknowledgements}

We deeply thank Menna M. Fouda, M.Sc., Oncology Department, Faculty of Medicine, Tanta University, for her great assistance in collecting the clinical and follow-up data from the patients' medical records.

\section{Authors' contributions}

$\mathrm{AE}$ and $\mathrm{NH}$ contributed equally in the proper selection of cases and their diagnosis. In addition, they had equal contribution in writing the manuscript, analysis, and interpretation of the patient data; regarding histopathological examination, immunohistochemical analysis, and assessment of results. All authors read and approved the final manuscript.

\section{Funding}

The authors declare that no funding was received to perform this study.

\section{Availability of data and materials}

The datasets used and/or analyzed during the current study are available from the corresponding author on reasonable request.

\section{Ethics approval and consent to participate}

The current study was conducted after obtaining the approval from research ethics committee, Faculty of Medicine, Tanta University, Egypt (reference\# 
34152). Informed written consent was obtained from involved participants in the study.

\section{Consent for publication}

Not applicable.

\section{Competing interests}

The authors declare that they have no competing interests.

Received: 14 October 2020 Accepted: 20 January 2021

Published online: 08 February 2021

\section{References}

1. Ellison DW, Dalton J, Kocak M, Nicholson SL, Fraga C, Neale G, et al. Medulloblastoma: clinicopathological correlates of $\mathrm{SHH}, \mathrm{WNT}$, and non-SHH/ WNT molecular subgroups. Acta Neuropathol. 2011;121(3):381-96.

2. Taylor MD, Northcott PA, Korshunov A, Remke M, Cho Y-J, Clifford SC, et al. Molecular subgroups of medulloblastoma: the current consensus. Acta Neuropathol. 2012;123(4):465-72.

3. Doussouki ME, Gajjar A, Chamdine O. Molecular genetics of medulloblastoma in children: diagnostic, therapeutic and prognostic implications. Future Neurol. 2019;14(1):FNL8.

4. Schwalbe EC, Lindsey JC, Nakjang S, Crosier S, Smith AJ, Hicks D, et al. Novel molecular subgroups for clinical classification and outcome prediction in childhood medulloblastoma: a cohort study. The Lancet Oncol. 2017;18(7): 958-71.

5. Pietsch T, Haberler C. Update on the integrated histopathological and genetic classification of medulloblastoma-a practical diagnostic guideline. Clin Neuropathol. 2016;35(11):344-52.

6. Cho Y-J, Tsherniak A, Tamayo P, Santagata S, Ligon A, Greulich H, et al. Integrative genomic analysis of medulloblastoma identifies a molecular subgroup that drives poor clinical outcome. J Clin Oncol. 2011;29(11):142430.

7. Tamayo P, Cho Y-J, Tsherniak A, Greulich H, Ambrogio L, Schouten-van Meeteren $\mathrm{N}$, et al. Predicting relapse in patients with medulloblastoma by integrating evidence from clinical and genomic features. J Clin Oncol. 2011; 29(11):1415-23.

8. Min HS, Lee YJ, Park K, Cho B-K, Park S-H. Medulloblastoma: histopathologic and molecular markers of anaplasia and biologic behavior. Acta neuropathol. 2006;112(1):13-20.

9. Mendoza PR, Specht CS, Hubbard GB, Wells JR, Lynn MJ, Zhang Q, et al. Histopathologic grading of anaplasia in retinoblastoma. Am J Ophthalmol. 2015;159(4):764-76.

10. Goschzik T, Zur Mühlen A, Kristiansen G, Haberler C, Stefanits H, Friedrich C, et al. Molecular stratification of medulloblastoma: comparison of histological and genetic methods to detect Wnt activated tumours. Neuropathol Appl Neurobiol. 2015;41(2):135-44.

11. Yu J, Shi W, Li H. Factors affecting the prognosis of children with medulloblastoma: a single institution retrospective analysis of 40 cases. Transl Neurosci Clin. 2017;3(1):16-27.

12. DeSouza R-M, Jones BR, Lowis SP, Kurian KM. Pediatric medulloblastomaupdate on molecular classification driving targeted therapies. Front Oncol. 2014;4(176):1-8

13. Jiang T, Zhang Y, Wang J, Du J, Qiu X, Wang Y, et al. A retrospective study of progression-free and overall survival in pediatric medulloblastoma based on molecular subgroup classification: A single-institution experience. Front Oncol. 2017:8:198.

14. Al-Halabi H, Nantel A, Klekner A, Guiot M-C, Albrecht S, Hauser P, et al. Preponderance of sonic hedgehog pathway activation characterizes adult medulloblastoma. Acta neuropathol. 2011;121(2):229-39.

15. Pietsch T, Schmidt R, Remke M, Korshunov A, Hovestadt $V$, Jones DT, et al. Prognostic significance of clinical, histopathological, and molecular characteristics of medulloblastomas in the prospective HIT2000 multicenter clinical trial cohort. Acta neuropathol. 2014;128(1):137-49.

16. Gibson P, Tong Y, Robinson G, Thompson MC, Currle DS, Eden C, et al. Subtypes of medulloblastoma have distinct developmental origins. Nature. 2010;468(7327):1095-9.

17. Northcott PA, Korshunov A, Pfister SM, Taylor MD. The clinical implications of medulloblastoma subgroups. Nat Rev Neurol. 2012;8(6):340-51.
18. Zhukova N, Ramaswamy V, Remke M, Pfaff E, Shih DJ, Martin DC, et al. Subgroup-specific prognostic implications of TP53 mutation in medulloblastoma. J Clin Oncol. 2013;31(23):2927.

19. Kool M, Korshunov A, Remke M, Jones DT, Schlanstein M, Northcott PA, et al. Molecular subgroups of medulloblastoma: an international metaanalysis of transcriptome, genetic aberrations, and clinical data of WNT, SHH, Group 3, and Group 4 medulloblastomas. Acta neuropathol. 2012; 123(4):473-84.

20. Tarbell NJ, Friedman H, Polkinghorn WR, Yock T, Zhou T, Chen Z, et al. Highrisk medulloblastoma: a pediatric oncology group randomized trial of chemotherapy before or after radiation therapy (POG 9031). J Clin Oncol. 2013:31(23):2936.

21. Sirachainan N, Nuchprayoon I, Thanarattanakorn P, Pakakasama S, Lusawat A, Visudibhan A, et al. Outcome of medulloblastoma in children treated with reduced-dose radiation therapy plus adjuvant chemotherapy. J Clin Neurosci. 2011;18(4):515-59.

22. Nalita N, Ratanalert S, Kanjanapradit K, Chotsampancharoen T, Tunthanathip T. Survival and prognostic factors in pediatric patients with medulloblastoma in southern Thailand. J Pediatr Neurosci. 2018;13(2):150-7.

23. Louis DN, Ohgaki H, Wiestler OD, Cavenee WK, Burger PC, Jouvet A, et al. The 2007 WHO classification of tumours of the central nervous system. Acta Neuropathol. 2007:114(2):97-109.

24. Gupta T, Dasgupta A, Epari S, Shirsat N, Chinnaswamy G, Jalali R. Extraneuraxial metastases in medulloblastoma: is histology and molecular biology important? J Neurooncol. 2017;135(2):419-21.

25. Giangaspero F, Wellek S, Masuoka J, Gessi M, Kleihues P, Ohgaki H. Stratification of medulloblastoma on the basis of histopathological grading. Acta Neuropathol. 2006;112(1):5-12.

26. Ramaswamy V, Remke M, Bouffet E, Bailey S, Clifford SC, Doz F, et al. Risk stratification of childhood medulloblastoma in the molecular era: the current consensus. Acta neuropathol. 2016;131(6):821-31.

27. Thompson EM, Hielscher T, Bouffet E, Remke M, Luu B, Gururangan S, et al. Prognostic value of medulloblastoma extent of resection after accounting for molecular subgroup: a retrospective integrated clinical and molecular analysis. Lancet Oncol. 2016;17(4):484-95.

28. Phoenix TN, Patmore DM, Boop S, Boulos N, Jacus MO, Patel YT, et al. Medulloblastoma genotype dictates blood brain barrier phenotype. Cancer cell. 2016;29(4):508-22.

29. Ramaswamy V, Remke M, Adamski J, Bartels U, Tabori U, Wang X, et al. Medulloblastoma subgroup-specific outcomes in irradiated children: who are the true high-risk patients? Neuro Oncol. 2016;18(2):291-7.

30. Juraschka K, Taylor MD. Medulloblastoma in the age of molecular subgroups: a review. JNSPG 75th Anniversary Invited Review Article. J Neurosurg Pediatr. 2019;24(4):353-63.

\section{Publisher's Note}

Springer Nature remains neutral with regard to jurisdictional claims in published maps and institutional affiliations.

\section{Submit your manuscript to a SpringerOpen ${ }^{\circ}$ journal and benefit from:}

- Convenient online submission

- Rigorous peer review

- Open access: articles freely available online

High visibility within the field

- Retaining the copyright to your article

Submit your next manuscript at $>$ springeropen.com 